Вестник КРАУНЦ. Физ.-мат. науки. 2020. Т. 32. № 3. С. 180-196. ISSN 2079-6641

MSC 35A08, 35J25, 35J70, 35J75

Research Article

\title{
Holmgren problem for elliptic equation with singular coefficients
}

\author{
T.G. Ergashev ${ }^{1,3}$, A. Hasanov 1,2
}

1 V.I. Romanovskiy Institute of Mathematics, Uzbekistan Academy of Sciences, M. Ulugbek str. 81, 100125, Tashkent, Uzbekistan

2 Department of Mathematics, Analysis, Logic and Discrete Mathematics of Ghent University, B 9000, Gent, Krijgslaan 281, Belgium

3 Institute of Irrigation and Agricultural Mechanization Engineers, 100100, Tashkent, Kari-Niyazi st., 39, Uzbekistan

E-mail: ergashev.tukhtasin@gmail.com

In the present work, we investigate the Holmgren problem for an multidimensional elliptic equation with several singular coefficients. We use a fundamental solution of the equation, containing Lauricella's hypergeometric function in many variables. Then using an "abc" method, the uniqueness for the solution of the Holmgren problem is proved. Applying a method of Green's function, we are able to find the solution of the problem in an explicit form. Moreover, decomposition and summation formulae, formulae of differentiation and some adjacent relations for Lauricella's hypergeometric functions in many variables were used in order to find the explicit solution for the formulated problem.

Keywords: Holmgren problem, multidimensional elliptic equations with several singular coefficients, decomposition formula, summation formula, Lauricella hypergeometric function in many variables, Green's function

DOI: $10.26117 / 2079-6641-2020-32-3-180-196$

Original article submitted: 04.07.2020

Revision submitted: 07.10 .2020

For citation. Ergashev T. G., Hasanov A. Holmgren problem for elliptic equation with singular coefficients. Vestnik KRAUNC. Fiz.-mat. nauki. 2020, 32: 3, 180-196. DOI: 10.26117/2079-66412020-32-3-180-196

The content is published under the terms of the Creative Commons Attribution 4.0 International License (https://creativecommons.org/licenses/by/4.0/deed.ru)

(C) Ergashev T. G., Hasanov A., 2020

\section{Introduction}

It is well-known that due to the many applications in aerodynamics [1] and irrigation problems [2], the theory of boundary value problems for singular partial differential equations (PDEs) has become a rapidly developing area in the general theory of PDEs. In addition, generalized axisymmetric potentials have been studied using various methods $[3,4,5]$.

Funding. This research received no specific grant from any funding agency in the public, commercial, or not-for-profit sectors 
Relatively few works are devoted to the study of boundary value problems for multidimensional (more two-dimensional) elliptic equations with singular coefficients.

Let $\mathrm{R}_{m}^{n+}$ be denote a $2^{n}$ th part of the $m$-dimensional Euclidean space:

$$
\mathrm{R}_{m}^{n+}:=\left\{\left(x_{1}, \ldots, x_{m}\right): x_{1}>0, \ldots, x_{n}>0,1 \leq n \leq m, m \geq 2\right\}
$$

In the present work we study the Holmgren problem for multidimensional elliptic equation with several singular coefficients

$$
H_{\alpha}^{(m, n)}(u) \equiv \sum_{i=1}^{m} \frac{\partial^{2} u}{\partial x_{i}^{2}}+\sum_{k=1}^{n} \frac{2 \alpha_{k}}{x_{k}} \frac{\partial u}{\partial x_{k}}=0
$$

in the domain lying in $\mathrm{R}_{m}^{n+}$, where $\alpha:=\left(\alpha_{1}, \ldots, \alpha_{n}\right) ; \quad \alpha_{k}$ are constants with $0<2 \alpha_{k}<$ $1, k \in \mathbb{K}, \mathbb{K}=\{1, \ldots, n\} ; n$ is a number of the singular coefficients of equation (1).

Holmgren problem is that in part of the boundary of the considered domain where the solution is sought, the values of the desired function are known, and in the remaining part of the boundary, the values of the derivative of the same function are given.

In 1926 Holmgren [6] posed a new problem for equation

$$
u_{x_{1} x_{1}}+u_{x_{2} x_{2}}+\frac{2 \alpha_{1}}{x_{1}} u_{x_{1}}=0
$$

in a finite domain lying in a half-plane $x_{1}>0$.

In the three-dimensional case [7] the solution of the Holmgren problem for equation

$$
u_{x_{1} x_{1}}+u_{x_{2} x_{2}}+u_{x_{3} x_{3}}+\frac{2 \alpha_{1}}{x_{1}} u_{x_{1}}+\frac{2 \alpha_{2}}{x_{2}} u_{x_{2}}+\frac{2 \alpha_{3}}{x_{3}} u_{x_{3}}=0
$$

in the first octant of the ball is written out through the Lauricella hypergeometric function in three variables $F_{A}^{(3)}$ (for definition, see a formula (4)).

The present paper is organized as follows: First, we give some preliminary information, which will be used in what follows. Second, we formulate the problem and prove a uniqueness theorem. In the rest of the paper handling the method of Green's function we find an explicit solution of the problem with the Holmgren conditions for equation (1). Finally, we state our main result as a theorem.

\section{Preliminaries}

Below we give some formulas for Euler gamma-function, Gauss hypergeometric function, multiple Lauricella hypergeometric function (that is, Lauricella hypergeometric function in several variables), which will be used in the next sections.

Let be $\mathbb{C}$ set of complex numbers and $\mathbb{N}$ set of the natural numbers : $\mathbb{N}=\{1,2,3, \ldots\}$.

It is known that the Euler gamma-function $\Gamma(a)$ has property [8, Ch.1, 1.2(2)]

$$
\Gamma(a+p)=\Gamma(a)(a)_{p}, a \in \mathbb{C}, p \in \mathbb{N} \cup\{0\} .
$$

Here $(a)_{p}$ is a Pochhammer symbol, for which the equality $(a)_{p+q}=(a)_{p}(a+p)_{q}$ is true.

A function

$$
F(a, b ; c ; y) \equiv F\left[\begin{array}{l}
a, b ; y \\
c ;
\end{array}\right]:=\sum_{p=0}^{\infty} \frac{(a)_{p}(b)_{p}}{(c)_{p} p !} y^{p},|y|<1, a, b, c, y \in \mathbb{C},
$$


is known as the Gauss hypergeometric function and an equality

$$
F(a, b ; c ; 1)=\frac{\Gamma(c) \Gamma(c-a-b)}{\Gamma(c-a) \Gamma(c-b)}[c \neq 0,-1,-2, \ldots ; \operatorname{Re}(c-a-b)>0]
$$

holds [8, Ch.2, 2.1(14)]. Moreover, the following autotransformer formula [8, Ch.2, $2.1(22)]$

$$
F(a, b ; c ; y)=(1-y)^{-b} F\left(c-a, b ; c ; \frac{y}{y-1}\right)
$$

is valid.

The Lauricella hypergeometric function in $n \in \mathbb{N}$ variables has a form $[9$, Ch.1, 1.4(1)]

$$
\begin{aligned}
F_{A}^{(n)} & \left(a, b_{1}, \ldots, b_{n} ; c_{1}, \ldots, c_{n} ; z_{1}, \ldots, z_{n}\right) \\
& \equiv F_{A}^{(n)}\left[\begin{array}{l}
\left.a, b_{1}, \ldots, b_{n} ; z_{1}, \ldots, z_{n}\right] \\
c_{1}, \ldots, c_{n} ;
\end{array}\right. \\
& =\sum_{m_{1}, \ldots m_{n}=0}^{\infty} \frac{(a)_{m_{1}+\ldots+m_{n}}\left(b_{1}\right)_{m_{1}} \ldots\left(b_{n}\right)_{m_{n}}}{\left(c_{1}\right)_{m_{1}} \ldots\left(c_{n}\right)_{m_{n}}} \frac{z_{1}^{m_{1}}}{m_{1} !} \ldots \frac{z_{n}^{m_{n}}}{m_{n} !} \\
& {\left[c_{k} \neq 0,-1,-2, \ldots ;\left|z_{1}\right|+\ldots+\left|z_{n}\right|<1 ; a, b_{k}, c_{k}, z_{k} \in \mathbb{C}\right] . }
\end{aligned}
$$

We give some elementary relations for $F_{A}^{(n)}$ necessary in this study:

$$
\begin{aligned}
& \frac{\partial}{\partial z_{k}} F_{A}^{(n)}\left(a, b_{1}, \ldots, b_{n} ; c_{1}, \ldots, c_{n} ; z_{1}, \ldots, z_{n}\right) \\
&=\frac{a b_{k}}{c_{k}} F_{A}^{(n)}\left[\begin{array}{l}
a+1, b_{1}, \ldots, b_{k-1}, b_{k}+1, b_{k+1}, \ldots, b_{n} ; \\
c_{1}, \ldots, c_{k-1}, c_{k}+1, c_{k+1}, \ldots, c_{n} ;
\end{array}, z_{1}, \ldots \in \mathbb{K},\right. \\
& \sum_{k=1}^{n} \frac{b_{k}}{c_{k}} z_{k} F_{A}^{(n)}\left[\begin{array}{l}
a+1, b_{1}, \ldots, b_{k-1}, b_{k}+1, b_{k+1}, \ldots, b_{n} ; \\
c_{1}, \ldots, c_{k-1}, c_{k}+1, c_{k+1}, \ldots, c_{n} ;
\end{array}\right] \\
&=F_{A}^{(n)}\left[\begin{array}{l}
a+1, b_{1}, \ldots, b_{n} ; z_{1}, \ldots, z_{n} \\
c_{1}, \ldots, c_{n} ;
\end{array}\right]-F_{A}^{(n)}\left[\begin{array}{l}
a, b_{1}, \ldots, b_{n} ; \\
c_{1}, \ldots, c_{n} ;
\end{array} z_{1}, \ldots, z_{n}\right] .
\end{aligned}
$$

Relations (5) and (6) can be proved in two ways: by comparing coefficients of equal powers of $z_{1}, \ldots, z_{n}$ on both sides or mathematical induction.

For a given multiple hypergeometric function, it is useful to fund a decomposition formula which would express the multivariable hypergeometric function in terms of products of several simpler hypergeometric functions involving fewer variables. Burchnall and Chaundy $[10,11]$ systematically presented a number of expansions and decomposition formulas for some double hypergeometric functions in series of simpler hypergeometric functions. Using the Burchnall-Chaundy method, Hasanov and Srivastava [12, 13] found decomposition formulas for a whole class of hypergeometric functions in several (three and more) variables. For example, the hypergeometric Lauricelli function $F_{A}^{(n)}$, defined 
by formula (4) has the Hasanov-Srivastava's decomposition formula [12]

$$
\begin{aligned}
F_{A}^{(n)} & \left(a, b_{1}, \ldots, b_{n} ; c_{1}, \ldots, c_{n} ; z_{1}, \ldots, z_{n}\right) \\
& =\sum_{m_{2}, \ldots, m_{n}=0}^{\infty} \frac{(a)_{m_{2}+\ldots+m_{n}}\left(b_{1}\right)_{m_{2}+\ldots+m_{n}}\left(b_{2}\right)_{m_{2}} \ldots\left(b_{n}\right)_{m_{n}}}{m_{2} ! \ldots m_{n} !\left(c_{1}\right)_{m_{2}+\ldots+m_{n}}\left(c_{2}\right)_{m_{2}} \ldots\left(c_{n}\right)_{m_{n}}} z_{1}^{m_{2}+\ldots+m_{n}} z_{2}^{m_{2}} \ldots z_{n}^{m_{n}} \\
& \times F\left(a+m_{2}+\ldots+m_{n}, b_{1}+m_{2}+\ldots+m_{n} ; c_{1}+m_{2}+\ldots+m_{n} ; z_{1}\right) \\
& \times F_{A}^{(n-1)}\left[\begin{array}{l}
a+m_{2}+\ldots+m_{n}, b_{2}+m_{2}, \ldots, b_{n}+m_{n} ; z_{2}, \ldots, z_{n} \\
c_{2}+m_{2}, \ldots, c_{n}+m_{n} ;
\end{array}\right], n \in \mathbb{N} \backslash\{1\} .
\end{aligned}
$$

However, due to the recurrence of formula (7), additional difficulties may arise in the applications of this expansion. Further study of the hypergeometric Lauricelli function $F_{A}^{(n)}$, showed that formula (7) can be reduced to a more convenient form.

Lemma 1 [14]. The following decomposition formula holds true at $n \in \mathbb{N} \backslash\{1\}$

$$
\begin{aligned}
F_{A}^{(n)} & \left(a, b_{1}, \ldots ., b_{n} ; c_{1}, \ldots ., c_{n} ; z_{1}, \ldots, z_{n}\right) \\
& =\sum_{\substack{m_{i, j}=0 \\
(2 \leq i \leq j \leq n)}}^{\infty} \frac{(a)_{A(n, n)}}{m_{i j} !} \prod_{k=1}^{n}\left[\frac{\left(b_{k}\right)_{B(k, n)}}{\left(c_{k}\right)_{B(k, n)}} z_{k}^{B(k, n)} F\left(\begin{array}{l}
a+A(k, n), b_{k}+B(k, n) ; \\
c_{k}+B(k, n) ;
\end{array}\right)\right],
\end{aligned}
$$

where

$$
A(k, n)=\sum_{i=2}^{k+1} \sum_{j=i}^{n} m_{i, j}, B(k, n)=\sum_{i=2}^{k} m_{i, k}+\sum_{i=k+1}^{n} m_{k+1, i} .
$$

Lemma 2. Let $a, b_{1}, \ldots, b_{n} \in \mathbb{C}$ be with $a \neq 0,-1,-2, \ldots$ and $\operatorname{Re}\left(a-b_{1}-\ldots-b_{n}\right)>0$. Then the following summation formula holds true at $n \in \mathbb{N} \backslash\{1\}$

$$
\sum_{\substack{m_{i, j}=0 \\(2 \leq i \leq j \leq n)}}^{\infty} \frac{(a)_{A(n, n)}}{m_{i j} !} \prod_{k=1}^{n} \frac{\left(b_{k}\right)_{B(k, n)}\left(a-b_{k}\right)_{A(k, n)-B(k, n)}}{(a)_{A(k, n)}}=\frac{\Gamma\left(a-\sum_{k=1}^{n} b_{k}\right)}{\prod_{k=1}^{n} \Gamma\left(a-b_{k}\right)} \Gamma^{n-1}(a),
$$

Formulae (8) and (10) are proved by the method mathematical induction [14].

Obviously, in the case when $n=2$ the formula (10) coincide with the summation formula (2).

The fundamental solutions of equation (1) in the domain $\mathrm{R}_{m}^{n+}$ were found in [14], one of which looks like:

$$
q(x ; \xi)=\gamma r^{-2 \bar{\alpha}} F_{A}^{(n)}\left(\bar{\alpha}, \alpha_{1}, \ldots, \alpha_{n} ; 2 \alpha_{1}, \ldots, 2 \alpha_{n} ; \sigma\right),
$$

where

$$
\begin{gathered}
x:=\left(x_{1}, \ldots, x_{m}\right), \xi:=\left(\xi_{1}, \ldots, \xi_{m}\right), \sigma:=\left(\sigma_{1}, \ldots, \sigma_{n}\right) \\
\gamma=2^{2 \bar{\alpha}-m} \frac{\Gamma(\bar{\alpha})}{\pi^{m / 2}} \prod_{k=1}^{n} \frac{\Gamma\left(\alpha_{k}\right)}{\Gamma\left(2 \alpha_{k}\right)}, \bar{\alpha}=\frac{m-2}{2}+\sum_{k=1}^{n} \alpha_{k}, 0<2 \alpha_{k}<1 \\
r^{2}=\sum_{i=1}^{m}\left(x_{i}-\xi_{i}\right)^{2}, r_{k}^{2}=\left(x_{k}+\xi_{k}\right)^{2}+\sum_{i=1, i \neq k}^{m}\left(x_{i}-\xi_{i}\right)^{2}, \sigma_{k}=1-\frac{r_{k}^{2}}{r^{2}}, k \in \mathbb{K} .
\end{gathered}
$$


It is easy to verify that the fundamental solution $q(x ; \xi)$ has the property

$$
\left.\left(x_{k}^{2 \alpha_{k}} \frac{\partial q(x ; \xi)}{\partial x_{k}}\right)\right|_{x_{k}=0}=0, k \in \mathbb{K} .
$$

\section{Formulation of the problem and the uniqueness of the solution}

Let $\Omega$ be a finite domain in $R_{m}^{n+}$, bounded by the Lyapunov surface $S$ [15] and the hyperplanes $x_{1}=0, \ldots, x_{n}=0$. The boundary of the domain $\Omega$ on the hyperplane $x_{k}=0$ is denoted by $S_{k}$.

We introduce the following notations:

$$
\begin{gathered}
x^{(2 \alpha)}:=x_{1}^{2 \alpha_{1}} \ldots x_{n}^{2 \alpha_{n}}, \tilde{x}_{k}^{(2 \alpha)}:=x_{1}^{2 \alpha_{1}} \ldots x_{k-1}^{2 \alpha_{k-1}} x_{k+1}^{2 \alpha_{k+1}} \ldots x_{n}^{2 \alpha_{n}} ; \\
\tilde{x}_{k}=\left(x_{1}, \ldots, x_{k-1}, x_{k+1}, \ldots, x_{m}\right) ; \tilde{x}_{k 0}=\left(x_{1}, \ldots, x_{k-1}, 0, x_{k+1}, \ldots, x_{m}\right) ; \\
d x=d x_{1} \ldots d x_{m} ; d \tilde{x}_{k}=d x_{1} \ldots d x_{k-1} d x_{k+1} \ldots d x_{m}, k \in \mathbb{K} .
\end{gathered}
$$

Holmgren problem. To find a function $u(x) \in C(\bar{\Omega}) \cap C^{2}(\Omega)$, satisfying equation (1) in $\Omega$ and conditions

$$
\begin{aligned}
\left.\left(x_{k}^{2 \alpha_{k}} \frac{\partial u}{\partial x_{k}}\right)\right|_{x_{k}=0} & =v_{k}\left(\tilde{x}_{k}\right), \quad \tilde{x}_{k} \in S_{k}, k \in \mathbb{K}, \\
\left.u\right|_{S} & =\varphi(x), x \in \bar{S},
\end{aligned}
$$

where $v_{k}\left(\tilde{x}_{k}\right)$ and $\varphi(x)$ are given functions, and, moreover, $v_{k}\left(\tilde{x}_{k}\right)$ can reduce to an infinity of the order less than $m-1-2 \alpha_{k}$ on the boundaries of $S_{k}$.

Uniqueness of the solution of the Holmgren problem. One can readily check the validity of the following relation

$$
x^{(2 \alpha)}\left[u H_{\alpha}^{(m, n)}(w)-w H_{\alpha}^{(m, n)}(u)\right]=\sum_{i=1}^{m} \frac{\partial}{\partial x_{i}}\left[x^{(2 \alpha)}\left(u \frac{\partial w}{\partial x_{i}}-w \frac{\partial u}{\partial x_{i}}\right)\right], n \leq m .
$$

Integrating both sides of the identity (17) in a domain $\Omega$ and using the GaussOstrogradsky formula, we obtain

$$
\int_{\Omega} x^{(2 \alpha)}\left[u H_{\alpha}^{(m, n)}(w)-w H_{\alpha}^{(m, n)}(u)\right] d x=\int_{S^{\prime}} x^{(2 \alpha)}\left(u \frac{\partial w}{\partial N}-w \frac{\partial u}{\partial N}\right) d S^{\prime},
$$

where $S^{\prime}$ is the boundary of $\Omega, N$ is the outer normal to $S^{\prime}$ and

$$
\frac{\partial[]}{\partial N}=\sum_{i=1}^{m} \frac{\partial[]}{\partial x_{i}} \cos \left(N, x_{i}\right)
$$

is the normal derivative with respect to $x$.

Assuming that $w=1$ and replacing $u$ by $u^{2}$ in (18), we obtain

$$
\int_{\Omega} x^{(2 \alpha)} \sum_{i=1}^{m}\left(\frac{\partial u}{\partial x_{i}}\right)^{2} d x=\sum_{k=1}^{n} \int_{S_{k}} \tilde{x}_{k}^{(2 \alpha)} \tau_{k}\left(\tilde{x}_{k}\right) v_{k}\left(\tilde{x}_{k}\right) d S_{k}-\int_{S} x^{(2 \alpha)} \varphi(x) \frac{\partial u}{\partial N} d S,
$$

where $u$ is a solution of equation (1) and $\tau_{k}\left(\tilde{x}_{k}\right):=\left.u\right|_{x_{k}=0}, k \in \mathbb{K}$. 
To prove the uniqueness of the solution, as usual, we suppose that the problem has two $v$ and $w$ solutions. Denoting $u=v-w$ we have that satisfies homogeneous Holmgren problem $\left(v_{k}=0, \varphi=0\right)$. Further we have to prove that the homogeneous problem has only trivial solution. In this case from (19) one can easily get

$$
\int_{\Omega} x^{(2 \alpha)} \sum_{i=1}^{m}\left(\frac{\partial u}{\partial x_{i}}\right)^{2} d x=0
$$

Hence, it follows that $u_{x_{1}}=\ldots=u_{x_{m}}=0$, which implies that $u$ is a constant function. Considering homogeneous condition (16), we conclude that $u(x) \equiv 0$ in $\bar{\Omega}$.

\section{The existence of the solution}

We prove the existence of the solution in a special case of the domain $\Omega$ in order to get the solution in an explicit form. Let $\Omega$ and $S$ be a $2^{n}$ th part of the multidimensional ball and sphere with the radius $a$ and center in the origin respectively:

$$
\begin{aligned}
& \Omega=\left\{x: x_{1}^{2}+\ldots+x_{m}^{2}<a^{2}, x_{1}>0, \ldots, x_{n}>0\right\}, \\
& S=\left\{x: x_{1}^{2}+\ldots+x_{m}^{2}=a^{2}, x_{1}>0, \ldots, x_{n}>0\right\} .
\end{aligned}
$$

We find a solution of considered problem using method Green's functions [16]. Therefore, first we give a definition of Green's function for the formulated problem.

Definition. We call the function $G(x ; \xi)$ as Green's function of the Holmgren problem, if it satisfies the following conditions:

this function is a regular solution of equation (1) in the domain $\Omega$, expect at the point $\xi$, which is any fixed point of $\Omega$;

it satisfies boundary conditions

$$
\left.\left(x_{k}^{2 \alpha_{k}} \frac{\partial G(x ; \xi)}{\partial x_{k}}\right)\right|_{x_{k}=0}=0, k \in \mathbb{K} ;\left.G(x ; \xi)\right|_{S}=0 ;
$$

it can be represented as

$$
G(x ; \xi)=q(x ; \xi)+q^{*}(x ; \xi),
$$

where $q(x ; \xi)$ is the fundamental solution found earlier (see a formula (11)), a function

$$
q^{*}(x ; \xi)=-\left(\frac{a}{R}\right)^{2 \bar{\alpha}} q(x ; \bar{\xi})
$$

is a regular solution of equation (1) in the domain $\Omega$. Here

$$
\bar{\xi}:=\left(\bar{\xi}_{1}, \ldots, \bar{\xi}_{m}\right), \quad \bar{\xi}_{i}=\frac{a^{2}}{R^{2}} \xi_{i}, a^{2}=x_{1}^{2}+\ldots+x_{m}^{2}, R^{2}=\xi_{1}^{2}+\ldots+\xi_{m}^{2}
$$

It is easy to see that a regular solution $q^{*}(x ; \xi)$ also has the property (14).

Excise a small ball with its center at $\xi$ and with radius $\rho>0$ from the domain $\Omega$. Designate the sphere of the excised ball as $C_{\rho}$ and by $\Omega_{\rho}$ denote the remaining part of $\Omega$. 
Applying formula (18), we obtain

$$
\begin{aligned}
\int_{C_{\rho}} x^{(2 \alpha)}[ & {\left[u(x) \frac{\partial G(x ; \xi)}{\partial N}-G(x ; \xi) \frac{\partial u(x)}{\partial N}\right] d C_{\rho} } \\
& =-\sum_{k=1}^{n} \int_{S_{k}} \tilde{x}_{k}^{(2 \alpha)} G\left(\tilde{x}_{k 0} ; \xi\right) v_{k}\left(\tilde{x}_{k}\right) d S_{k}-\int_{S} x^{(2 \alpha)} \frac{\partial G(x ; \xi)}{\partial N} \varphi(x) d S
\end{aligned}
$$

where

$$
S_{k}=\left\{x: \sum_{i=1, i \neq k}^{m} x_{i}^{2}=a^{2}, x_{1}>0, \ldots, x_{k-1}>0, x_{k+1}>0, \ldots, x_{n}>0\right\}, k \in \mathbb{K} .
$$

First, we consider an integral

$$
\int_{C_{\rho}} x^{(2 \alpha)} u(x) \frac{\partial G(x ; \xi)}{\partial N} d C_{\rho}
$$

Taking (20) into account we rewrite it as follows

$$
\begin{aligned}
\int_{C_{\rho}} x^{(2 \alpha)} u(x) \frac{\partial G}{\partial N} d C_{\rho} & \equiv \int_{C_{\rho}} x^{(2 \alpha)} u(x) \frac{\partial q(x ; \xi)}{\partial N} d C_{\rho}+\int_{C_{\rho}} x^{(2 \alpha)} u(x) \frac{\partial q^{*}(x ; \xi)}{\partial N} d C_{\rho} \\
& =I_{1}(\xi, \rho)+I_{2}(\xi, \rho) .
\end{aligned}
$$

Using the formula of differentiation (5) and the adjacent relation (6), we calculate

$$
\frac{\partial q(x ; \xi)}{\partial N}=\sum_{i=1}^{m} \frac{\partial q(x ; \xi)}{\partial x_{i}} \cdot \cos \left(N, x_{i}\right)
$$

Below we get detailed evaluations for $\frac{\partial q(x ; \xi)}{\partial x_{i}}$, when $1 \leq i \leq n$. Indeed, using the formula of differentiation (5), we get

$$
\begin{aligned}
\frac{\partial q(x, \xi)}{\partial x_{i}}= & -2 \bar{\alpha} \gamma\left(x_{i}-\xi_{i}\right) r^{-2 \bar{\alpha}-2} F_{A}^{(n)}\left[\begin{array}{l}
\bar{\alpha}, \alpha_{1}, \ldots, \alpha_{n} ; \\
2 \alpha_{1}, \ldots, 2 \alpha_{n} ;
\end{array}\right] \\
& -2 \bar{\alpha} \gamma\left(x_{i}-\xi_{i}\right) r^{-2 \bar{\alpha}-2} \sum_{k=1}^{n} \frac{\alpha_{k}}{2 \alpha_{k}} \sigma_{k} F_{A}^{(n)}\left[\begin{array}{l}
1+\bar{\alpha}, \alpha_{1}, \ldots, \alpha_{k-1}, 1+\alpha_{k}, \alpha_{k+1}, \ldots, \alpha_{n} ; \sigma \\
2 \alpha_{1}, \ldots, 2 \alpha_{k-1}, 1+2 \alpha_{k}, 2 \alpha_{k+1}, \ldots, 2 \alpha_{n} ;
\end{array}\right] \\
& -2 \bar{\alpha} \gamma \xi_{i} r^{-2 \bar{\alpha}-2} F_{A}^{(n)}\left[\begin{array}{l}
1+\bar{\alpha}, \alpha_{1}, \ldots, \alpha_{i-1}, 1+\alpha_{i}, \alpha_{i+1}, \ldots, \alpha_{n} ; \\
2 \alpha_{1}, \ldots, 2 \alpha_{i-1}, 1+2 \alpha_{i}, 2 \alpha_{i+1}, \ldots, 2 \alpha_{n} ;
\end{array}\right] .
\end{aligned}
$$

Considering adjacent relation (6) we obtain

$$
\begin{aligned}
\frac{\partial q(x, \xi)}{\partial x_{i}}= & -2 \bar{\alpha} \gamma\left(x_{i}-\xi_{i}\right) r^{-2 \bar{\alpha}-2} F_{A}^{(n)}\left[\begin{array}{l}
1+\bar{\alpha}, \alpha_{1}, \ldots, \alpha_{n} ; \\
2 \alpha_{1}, \ldots, 2 \alpha_{n} ;
\end{array}\right] \\
& -2 \bar{\alpha} \gamma \xi_{i} r^{-2 \bar{\alpha}-2} F_{A}^{(n)}\left[\begin{array}{l}
1+\bar{\alpha}, \alpha_{1}, \ldots, \alpha_{i-1}, 1+\alpha_{i}, \alpha_{i+1}, \ldots, \alpha_{n} ; \\
2 \alpha_{1}, \ldots, 2 \alpha_{i-1}, 1+2 \alpha_{i}, 2 \alpha_{i+1}, \ldots, 2 \alpha_{n} ;
\end{array}\right], 1 \leq i \leq n .
\end{aligned}
$$

Similarly, we calculate

$$
\frac{\partial q(x, \xi)}{\partial x_{i}}=-2 \bar{\alpha} \gamma\left(x_{i}-\xi_{i}\right) r^{-2 \bar{\alpha}-2} F_{A}^{(n)}\left[\begin{array}{l}
1+\bar{\alpha}, \alpha_{1}, \ldots, \alpha_{n} ; \\
2 \alpha_{1}, \ldots, 2 \alpha_{n} ;
\end{array}\right], n+1 \leq i \leq m .
$$


Taking (22), (23) and (24) into account we calculate

$$
\begin{aligned}
\frac{\partial q(x, \xi)}{\partial N}= & -\bar{\alpha} \gamma r^{-2 \bar{\alpha}} F_{A}^{(n)}\left[\begin{array}{l}
\left.1+\bar{\alpha}, \alpha_{1}, \ldots, \alpha_{n} ; \sigma\right] \\
2 \alpha_{1}, \ldots, 2 \alpha_{n} ;
\end{array}\right] \frac{\partial}{\partial N}\left[\ln r^{2}\right] \\
& -2 \bar{\alpha} \gamma r^{-2 \bar{\alpha}-2} \sum_{k=1}^{n} \xi_{k} F_{A}^{(n)}\left[\begin{array}{l}
1+\bar{\alpha}, \alpha_{1}, \ldots, \alpha_{k-1}, 1+\alpha_{k}, \alpha_{k+1}, \ldots, \alpha_{n} ; \\
2 \alpha_{1}, \ldots, 2 \alpha_{k-1}, 1+2 \alpha_{k}, 2 \alpha_{k+1}, \ldots, 2 \alpha_{n} ;
\end{array}\right] \cos \left(N ; x_{k}\right) .
\end{aligned}
$$

Now consider the integral

$$
\int_{C_{\rho}} x^{(2 \alpha)} u(x) \frac{\partial q(x ; \xi)}{\partial N} d C_{\rho}=I_{11}(\xi, \rho)+I_{12}(\xi, \rho),
$$

where

$$
\begin{aligned}
I_{11}(\xi, \rho)= & -\bar{\alpha} \gamma \int_{C_{\rho}} u(x) r^{-2 \bar{\alpha}} F_{A}^{(n)}\left[\begin{array}{l}
1+\bar{\alpha}, \alpha_{1}, \ldots, \alpha_{n} ; \\
2 \alpha_{1}, \ldots, 2 \alpha_{n} ;
\end{array}\right] \frac{\partial}{\partial N}\left[\ln r^{2}\right] d C_{\rho}, \\
I_{12}(\xi, \rho)= & -2 \bar{\alpha} \gamma \int_{C_{\rho}} u(x) r^{-2-2 \bar{\alpha}} \\
& \times \sum_{k=1}^{n} \xi_{k} F_{A}^{(n)}\left[\begin{array}{l}
\left.1+\bar{\alpha}, \alpha_{1}, \ldots, \alpha_{k-1}, 1+\alpha_{k}, \alpha_{k+1}, \ldots, \alpha_{n} ; \sigma\right] \cos \left(N ; x_{k}\right) d C_{\rho} . \\
2 \alpha_{1}, \ldots, 2 \alpha_{k-1}, 1+2 \alpha_{k}, 2 \alpha_{k+1}, \ldots, 2 \alpha_{n} ;
\end{array}\right]
\end{aligned}
$$

We use the following generalization spherical system of coordinates:

$$
x_{i}=\xi_{i}+\rho \Phi_{i}(\varphi), i=1, \ldots, m
$$

where

$$
\begin{gathered}
\varphi=\left(\varphi_{1}, \ldots, \varphi_{m-1}\right) ; \Phi_{1}(\varphi)=\cos \varphi_{1}, \\
\Phi_{i}(\varphi)=\sin \varphi_{1} \sin \varphi_{2} \cdots \sin \varphi_{i-1} \cos \varphi_{i}, i=2, \ldots, m-1, \\
\Phi_{m}(\varphi)=\sin \varphi_{1} \sin \varphi_{2} \cdots \sin \varphi_{m-2} \sin \varphi_{m-1} \\
\left(0 \leqq \rho \leqq r, 0 \leqq \varphi_{1} \leqq \pi, \ldots, 0 \leqq \varphi_{m-2} \leqq \pi, 0 \leqq \varphi_{m-1} \leqq 2 \pi\right)
\end{gathered}
$$

Then we have

$$
\begin{aligned}
I_{11}(\xi, \rho) & =2 \bar{\alpha} \gamma \rho^{-2 \alpha_{1}-\ldots-2 \alpha_{n}} \int_{0}^{2 \pi} d \varphi_{m-1} \int_{0}^{\pi} \sin \varphi_{m-2} d \varphi_{m-2} \\
& \times \int_{0}^{\pi} \sin ^{2} \varphi_{m-3} d \varphi_{m-3} \ldots \int_{0}^{\pi} u\left(\xi_{1}+\rho \Phi_{1}(\varphi), \ldots, \xi_{m}+\rho \Phi_{m}(\varphi)\right) \\
& \times \prod_{k=1}^{n}\left[\xi_{k}+\rho \Phi_{k}(\varphi)\right]^{2 \alpha_{k}} F_{A}^{(n)}\left[\begin{array}{c}
1+\bar{\alpha}, \alpha_{1}, \ldots, \alpha_{n} ; \\
2 \alpha_{1}, \ldots, 2 \alpha_{n} ;
\end{array}(\rho, \varphi)\right] \sin ^{m-2} \varphi_{1} d \varphi_{1},
\end{aligned}
$$

where

$$
\sigma(\rho, \varphi)=\left(1-\frac{r_{1 \rho}^{2}}{\rho^{2}}, \ldots, 1-\frac{r_{n \rho}^{2}}{\rho^{2}}\right), r_{k \rho}^{2}=4 \xi_{k}^{2}+4 \rho \xi_{k} \Phi_{k}(\varphi)+\rho^{2}
$$

First we evaluate a Lauricella's hypergeometric function in (25):

$$
F_{A}^{(n)}(\rho, \varphi):=F_{A}^{(n)}\left[\begin{array}{c}
1+\bar{\alpha}, \alpha_{1}, \ldots, \alpha_{n} ; \\
2 \alpha_{1}, \ldots, 2 \alpha_{n} ;
\end{array} \sigma(\rho, \varphi)\right] .
$$


Using the decomposition formula (8) and then auto-transformation formula (3), we rewrite a formula (26) as follows

$$
F_{A}^{(n)}(\rho, \varphi)=\rho^{2 \alpha_{1}+\ldots+2 \alpha_{n}} \prod_{k=1}^{n}\left[r_{k \rho}^{-2 \alpha_{k}}\right] \cdot P(\rho, \varphi),
$$

where

$$
\begin{aligned}
P(\rho, \varphi)= & \sum_{\substack{m_{i, j}=0 \\
(2 \leq i \leq j \leq n)}}^{\infty} \frac{(1+\bar{\alpha})_{A(n, n)}}{m_{i j} !} \prod_{k=1}^{n} \frac{\left(\alpha_{k}\right)_{B(k, n)}}{\left(2 \alpha_{k}\right)_{B(k, n)}}\left(\frac{\rho^{2}}{r_{k \rho}^{2}}-1\right)^{B(k, n)} \\
& \times \prod_{k=1}^{n} F\left(\begin{array}{c}
2 \alpha_{k}-\bar{\alpha}-1+B(k, n)-A(k, n), \alpha_{k}+B(k, n) ; 1-\frac{\rho^{2}}{r_{k \rho}^{2}} \\
2 \alpha_{k}+B(k, n) ;
\end{array}\right.
\end{aligned}
$$

where $A(k, n)$ and $B(k, n)$ are expressions defined in (9).

It is easy to see that when $\rho \rightarrow 0$ the function $P$ becomes an expression that does not depend on $\varphi$. Applying the summation formula (2) to each hypergeometric function $F(a, b ; c ; 1)$ in the sum $(28)$, we get

$$
\begin{aligned}
\lim _{\rho \rightarrow 0} P(\rho, \varphi)= & \frac{1}{\Gamma^{n}(1+\bar{\alpha})} \prod_{k=1}^{n} \frac{\Gamma\left(2 \alpha_{k}\right) \Gamma\left(1+\bar{\alpha}-\alpha_{k}\right)}{\Gamma\left(\alpha_{k}\right)} \\
& \times \sum_{\substack{m_{i, j}=0 \\
(2 \leq i \leq j \leq n)}}^{\infty} \frac{(1+\bar{\alpha})_{A(n, n)}}{m_{i j} !} \prod_{k=1}^{n} \frac{\left(\alpha_{k}\right)_{B(k, n)}\left(1+\bar{\alpha}-\alpha_{k}\right)_{A(k, n)-B(k, n)}}{(1+\bar{\alpha})_{A(k, n)}} .
\end{aligned}
$$

Taking into account the identity (10), we obtain

$$
\lim _{\rho \rightarrow 0} P(\rho, \varphi)=\frac{\Gamma(m / 2)}{\Gamma(1+\bar{\alpha})} \prod_{i=1}^{n} \frac{\Gamma\left(2 \alpha_{k}\right)}{\Gamma\left(\alpha_{k}\right)} .
$$

If we take into account (25), (27), (29) and (12), then we will have

$$
\lim _{\rho \rightarrow 0} I_{11}(\xi, \rho)=u(\xi)
$$

By similar evaluations one can get that

$$
\lim _{\rho \rightarrow 0} I_{12}(\xi, \rho)=\lim _{\rho \rightarrow 0} I_{2}(\xi, \rho)=0 .
$$

If we consider an integral

$$
\int_{C_{\rho}} x^{(2 \alpha)} G(x ; \xi) \frac{\partial u(x)}{\partial N} d C_{\rho}
$$

using above given algorithm for evaluations (in this case calculations will be more simple), we can prove that

$$
\lim _{\rho \rightarrow 0} \int_{C_{\rho}} x^{(2 \alpha)} G(x ; \xi) \frac{\partial u(x)}{\partial N} d C_{\rho}=0 .
$$


Now, by virtue of (30), (31) and (32), from (21) one can easily get a solution of the Holmgren problem in the following form:

$$
\begin{aligned}
& u(\xi)=-\gamma \sum_{k=1}^{n} \int_{S_{k}} \tilde{x}_{k}^{(2 \alpha)}\left\{X_{k}^{-2 \bar{\alpha}} F_{A}^{(n-1)}\left[\begin{array}{l}
\bar{\alpha}, \alpha_{1}, \ldots, \alpha_{k-1}, \alpha_{k+1}, \ldots, \alpha_{n} ; \\
2 \alpha_{1}, \ldots, 2 \alpha_{k-1}, 2 \alpha_{k+1}, \ldots, 2 \alpha_{n} ; \sigma_{k 0}
\end{array}\right]\right. \\
& \left.-Y_{k}^{-2 \bar{\alpha}} F_{A}^{(n-1)}\left[\begin{array}{l}
\bar{\alpha}, \alpha_{1}, \ldots, \alpha_{k-1}, \alpha_{k+1}, \ldots, \alpha_{n} ; \\
2 \alpha_{1}, \ldots, 2 \alpha_{k-1}, 2 \alpha_{k+1}, \ldots, 2 \alpha_{n} ;
\end{array} \bar{\sigma}_{k 0}\right]\right\} v_{k}\left(\tilde{x}_{k}\right) d \tilde{x}_{k} \\
& +2 \bar{\alpha} \gamma \int_{S} x^{(2 \alpha)} F_{A}^{(n)}\left[\begin{array}{c}
1+\bar{\alpha}, \alpha_{1}, \ldots, \alpha_{n} ; \\
2 \alpha_{1}, \ldots, 2 \alpha_{n} ;
\end{array}\right] \frac{R^{2}-a^{2}}{R r^{2+2 \bar{\alpha}}} \varphi(x) d S,
\end{aligned}
$$

where

$$
\begin{gathered}
\sigma_{k 0}:=\left(\sigma_{1}^{0}, \ldots, \sigma_{k-1}^{0}, \sigma_{k+1}^{0}, \ldots, \sigma_{n}^{0}\right), \quad \bar{\sigma}_{k 0}:=\left(\bar{\sigma}_{1}^{0}, \ldots, \bar{\sigma}_{k-1}^{0}, \bar{\sigma}_{k+1}^{0}, \ldots, \bar{\sigma}_{n}^{0}\right) \\
\sigma_{s}^{0}=-\frac{4 x_{s} \xi_{s}}{X_{k}^{2}}, \bar{\sigma}_{s}^{0}=-\frac{a^{2}}{R^{2}} \frac{4 x_{s} \xi_{s}}{Y_{k}^{2}}, s=1, \ldots, n, s \neq k \\
a^{2}=\sum_{i=1}^{m} x_{i}^{2}, R^{2}=\sum_{i=1}^{m} \xi_{i}^{2}, X_{k}^{2}=\xi_{k}^{2}+\sum_{i=1, i \neq k}^{m}\left(\xi_{i}-x_{i}\right)^{2} \\
Y_{k}^{2}=\sum_{i=1, i \neq k}^{m}\left(a-\frac{x_{i} \xi_{i}}{a}\right)^{2}+\frac{1}{a^{2}} \sum_{i=1, i \neq k}^{m} \sum_{j=1, j \neq i}^{m} x_{i}^{2} \xi_{j}^{2}-(m-2) a^{2}, k \in \mathbb{K} .
\end{gathered}
$$

Here constants $\bar{\alpha}, \gamma$ and variables $\sigma:=\left(\sigma_{1}, \ldots, \sigma_{n}\right), r^{2}$ are defined in (12) and (13), respectively.

The formula (33), and with it all the proof, requires that $m>2$. However, the formula (33) is also valid for $m=2$.

Hence, the main result of the paper is formulated as the following theorem:

Tеорема. If $v_{k}\left(\tilde{x}_{k}\right) \in C^{2}\left(S_{k}\right), k \in \mathbb{K}$ and $\varphi(x) \in C^{2}(S)$ are given functions, then the Holmgren problem with conditions (15) and (16) for equation (1) has unique solution represented by formula (33).

Competing interests. The authors declare that there are no conflicts of interest regarding authorship and publication.

Contribution and Responsibility. All authors contributed to this article. Authors are solely responsible for providing the final version of the article in print. The final version of the manuscript was approved by all authors.

\section{References}

[1] Bers L., Mathematical aspects of subsonic and transonic gas dynamics, New York. London, 1958.

[2] Serbina L. I., "A problem for the linearized Boussinesq equation with a nonlocal Samarskii condition", Differential Equations, 38(8) (2002), 1187-1194.

[3] Ergashev T. G., "Third double-layer potential for a generalized bi-axially symmetric Helmholtz equation", Ufa Mathematical Journal, 10(4) (2018), 111-122.

[4] Srivastava H. M., Hasanov A., Choi J., "Double-Layer Potentials for a Generalized BiAxially Symmetric Helmholtz Equation", Sohag Journal of Mathematics, 2(1) (2015), 1-10.

[5] Weinstein A., "Generalized axially symmetric potentials theory", Bull. Amer. Math. Soc., 59 (1959), 20-38. 
[6] Holmgren E., "Sur un probleme aux limites pour l'equation $y^{m} u_{x x}+u_{y y}=0$ ", Arkiv for matematik, astronomi och Fysik, 19B(14) (1926), 1-3.

[7] Karimov E. T., "On a boundary problem for 3-D elliptic equation with singular coefficients", Progress in Analysis and Its Applications, Proceeding of the 7th International ISAAC Congress, 13-18 July 2009, Imperial College London, UK, 2009, 619-625.

[8] Erdélyi A., Magnus W., Oberhettinger F., Tricomi F.G., Higher Transcendental Functions. V. I, McGraw-Hill Book Company, New York, Toronto and London, 1953.

[9] Srivastava H. M., Karlsson P. W., Multiple Gaussian Hypergeometric Series, Halsted Press, New York, Chichester, Brisbane and Toronto, 1985, 428 pp.

[10] Burchnall J. L., Chaundy T. W., "Expansions of Appell's double hypergeometric functions", The Quarterly Journal of Mathematics, 11 (1940), 249-270.

[11] Burchnall J. L., Chaundy T. W., "Expansions of Appell's double hypergeometric functions. II", The Quarterly Journal of Mathematics, 12 (1941), 112-128.

[12] Hasanov A., Srivastava H. M., "Some decomposition formulas associated with the Lauricella function $F_{A}^{(r)}$ and other multiple hypergeometric functions", Appl. Math. Lett., 19(2) (2006), $113-121$.

[13] Hasanov A., Srivastava H. M., "Decomposition Formulas Associated with the Lauricella Multivariable Hypergeometric Functions", Computers and Mathematics with Applications, 53(7) (2007), 1119-1128.

[14] Ergashev T. G., "Fundamental solutions for a class of multidimensional elliptic equations with several singular coefficients", Journal of Siberian Federal University. Mathematics and Physics, 13(1) (2020), 48-57.

[15] Mikhlin S. G., An Advanced Course of Mathematical Physics. North Holland Series in Applied Mathematics and Mechanics. V.11 Amsterdam, London, North-Holland Publishing.

[16] Rassias M., Lecture Notes on Mixed Type Partial Differential Equations, World Scientific, 1990.

\section{References (GOST)}

[1] Bers L. Mathematical aspects of subsonic and transonic gas dynamics. New York. London, 1958.

[2] Serbina L. I. A problem for the linearized Boussinesq equation with a nonlocal Samarskii condition // Differential Equations. 2002. vol. 38(8). pp. 1187-1194.

[3] Ergashev T.G. Third double-layer potential for a generalized bi-axially symmetric Helmholtz equation // Ufa Mathematical Journal. 2018. vol. 10(4). pp. 111-122.

[4] Srivastava H. M., Hasanov A., Choi J. Double-Layer Potentials for a Generalized Bi-Axially Symmetric Helmholtz Equation // Sohag Journal of Mathematics. 2015. vol. 2(1). pp. 1-10.

[5] Weinstein A. Generalized axially symmetric potentials theory // Bull. Amer. Math. Soc. 1959. vol. 59. pp. 20-38.

[6] Holmgren E. Sur un probleme aux limites pour l'equation $y^{m} u_{x x}+u_{y y}=0 / /$ Arkiv for matematik, astronomi och Fysik. 1926. 19B(14). pp. 1-3.

[7] Karimov E.T. On a boundary problem for 3-D elliptic equation with singular coefficients. Progress in Analysis and Its Applications. Proceeding of the 7 th International ISAAC Congress, Imperial College London, UK, 13-18 July 2009. pp. 619-625.

[8] Erdélyi A., Magnus W., Oberhettinger F., Tricomi F.G. Higher Transcendental Functions. vol. I. New York, Toronto and London: McGraw-Hill Book Company, 1953.

[9] Srivastava H. M., Karlsson P. W. Multiple Gaussian Hypergeometric Series. New York, Chichester, Brisbane and Toronto: Halsted Press, 1985. 428 p.

[10] Burchnall J.L., Chaundy T. W. Expansions of Appell's double hypergeometric functions // The Quarterly Journal of Mathematics. 1940. Ser. 11. pp. 249-270.

[11] Burchnall J. L., Chaundy T. W. Expansions of Appell's double hypergeometric functions. II // The Quarterly Journal of Mathematics. 1941. Ser. 12. pp. 112-128. 
[12] Hasanov A., Srivastava H. M. Some decomposition formulas associated with the Lauricella function $F_{A}^{(r)}$ and other multiple hypergeometric functions // Appl. Math. Lett. 2006. vol. 19(2). pp. 113-121.

[13] Hasanov A., Srivastava H. M. Decomposition Formulas Associated with the Lauricella Multivariable Hypergeometric Functions // Computers and Mathematics with Applications. 2007. vol. 53(7). pp. 1119-1128.

[14] Ergashev T.G. Fundamental solutions for a class of multidimensional elliptic equations with several singular coefficients // Journal of Siberian Federal University. Mathematics and Physics. 2020. vol. 13(1). pp. 48-57.

[15] Mikhlin S. G. An Advanced Course of Mathematical Physics. North Holland Series in Applied Mathematics and Mechanics, V. 11. Amsterdam, London: North-Holland Publishing, 1970.

[16] Rassias M. Lecture Notes on Mixed Type Partial Differential Equations. World Scientific, 1990. 


\title{
Задача Холмгрена для эллиптического уравнения с сингулярными коэффициентами
}

\author{
T. Г. Эргашев ${ }^{1,3}$, А. Хасанов ${ }^{1,2}$
}

1 Институт Математики имени В. И. Романовского Академии наук Узбекистана, г. Ташкент, ул. Мирзо Улугбека 85, 100170, Республика Узбекистан

2 Кафедра математики, анализа, логики и дискретной математики Гентского университета, г. Гент, Бельгия

3 Ташкентский институт инженеров ирригации и механизации сельского хозяйства, 100100, г. Ташкент, ул. Кари-Ниязи, 39, Республика Узбекистан

E-mail: ergashev.tukhtasin@gmail.com

В данной работе мы исследуем задачу Холмгрена для многомерного эллиптического уравнения с несколькими сингулярными коэффициентами. Мы используем фундаментальное решение уравнения, содержащее гипергеометрическую функцию Лауричеллы от многих переменных. Затем методом «abc» доказывается единственность решения проблемы Холмгрена. Применяя метод функции Грина, мы можем найти решение задачи в явном виде. Более того, формулы разложения и суммирования, формулы дифференцирования и некоторые смежные соотношения для гипергеометрических функций Лауричеллы от многих переменных были использованы для нахождения явного решения поставленной задачи.

Ключевые слова: задача Холмгрена, многомерные эллиптические уравнения с несколькими сингулярными коэффициентами, формула разложения, формула суммирования, гипергеометрическая функция Лауричеллы от многих переменных, функция Грина.

DOI: $10.26117 / 2079-6641-2020-32-3-180-196$

Поступила в редакцию: 04.07.2020

В окончательном варианте: 10.10 .2020

Для цитирования. Ergashev T. G., Hasanov A. Holmgren problem for elliptic equation with singular coefficients // Вестник КРАУНЦ. Физ.-мат. науки. 2020. Т. 32. № 3. С. 180-196. DOI: $10.26117 / 2079-6641-2020-32-3-180-196$

Конкурирующие интересы. Авторы заявляют, что конфликтов интересов в отношении авторства и публикации нет.

Авторский вклад и ответсвенность. Все авторы участвовали в написании статьи и полностью несут ответственность за предоставление окончательной версии статьи в печать. Окончательная версия рукописи была одобрена всеми авторами.

Контент публикуется на условиях лищензии Creative Commons Attribution 4.0 International (https://creativecommons.org/licenses/by/4.0/deed.ru)

(C) Ergashev T. G., Hasanov A., 2020

Финансирование. Исследование выполнялось без финансирования 